\title{
Modelos de política cultural y modelos de equipa- mientos culturales: de los modelos nacionales a los modelos locales. Análisis del caso de Barcelona
}

\author{
Joaquim RIUS ULLDEMOLINS \\ Universitat de València \\ joaquim.rius@uv.es
}

Recibido: 14-05-2013

Aceptado: 18-04-2014

\begin{abstract}
Resumen:
El desarrollo del análisis de la política cultural por parte de las ciencias sociales ha producido una teorización sobre los modelos de política cultural por parte de la sociología y la ciencia política. En estos análisis se plantea la influencia del modelo nacional de política cultural sobre las formas de gobernanza y gestión de los equipamientos culturales. No obstante, en este artículo defenderemos que actualmente es el modelo local de política cultural el que influye en el modelo de institución cultural. Ello se explica por la creciente importancia de la cultura en las estrategias de desarrollo local. Para demostrarlo analizaremos el caso del Modelo Barcelona de desarrollo local y política cultural, que destaca por el nivel de liderazgo del gobierno local, la gobernanza multinivel, el uso de la cultura en los procesos de planificación urbana y una tendencia a la concertación público-privado en la gestión pública. Un Modelo Barcelona que determinará la génesis y desarrollo de sus equipamientos culturales, generando un modelo singular y relativamente exitoso.
\end{abstract}

Palabras clave: Política cultural, Modelo Barcelona, equipamientos culturales, gobernanza cultural, cooperación público-privada 


\title{
Cultural policy models and cultural facilities models: from national to local models. Analysis of Barcelona's case
}

\begin{abstract}
The development of cultural policy analysis by social science has been produced a theorization about cultural policy models from sociology and political science. This analysis shows the influence of the national model of cultural policy on the forms of governance and management of cultural facilities. However, in this paper we will defend that currently the local model of cultural policy decisively influences the model of cultural institutions. This is explained by the growing importance of culture in local development strategies. In order to demonstrate this we will analyze the case of the Barcelona Model of local development and cultural policy, that is characterized for the level of local government leadership, multilevel governance, the use of culture in urban planning processes and a tendency to use public-private partnership in public management. This Model influences the genesis and development of the cultural facilities and it produces a singular and relatively successful model
\end{abstract}

Keywords: cultural policy, Barcelona Model, cultural facilities, cultural governance, public-private partnership

\section{Referencia normalizada}

Rius Ulldemolins, J. (2014). "Modelos de política cultural y modelos de equipamientos culturales: de los modelos nacionales a los modelos locales. Análisis del caso de Barcelona”. Política y Sociedad, Vol 51, Núm. 2: 399-422

Sumario: 1.Introducción. 2.Modelos de políticas culturales y tipos de equipamientos culturales: de los modelos nacionales a los modelos locales. 3.Modelo de política cultural y modelo de equipamientos culturales: el caso de barcelona. 4.Conclusiones. Bibliografía. 


\section{Introducción}

Desde los años ochenta se ha venido destacando de forma creciente la importancia de la cultura para el desarrollo de las ciudades, por su capacidad de favorecer el paso a la llamada nueva economía, por la posibilidad de estimular el surgimiento de clústeres de industrias creativas o su potencial para catalizar procesos de regeneración urbana (Bianchini, 1993a, Scott, 2000, Scott, 2010). Desde entonces, las políticas culturales se han transformado de forma notable incorporando esos objetivos de desarrollo económico y social (Gray, 2007), hecho que ha alterado las relaciones tradicionales entre la esfera cultural y política al introducir la necesidad de demostrar el impacto beneficioso del gasto público en cultura (Belfiore, 2004, Belfiore y Bennett, 2007). Ello ha motivado el surgimiento de lo que Gattinger y Saint Pierre (2008) han llamado modelos de política cultural híbridos, por ser una combinación de los modelos europeo-continental y liberal, y por ser un modelo en el que los niveles de gobierno regional y local tienen un rol mucho más activo que en etapas precedentes.

Este giro local y emprendedor de la política cultural (Rodriguez Morató, 2005) ha tenido en los grandes equipamientos culturales uno de sus principales instrumentos, bien por su capacidad de impulsar procesos de regeneración urbana (Whitt, 1987), bien por potenciar la imagen de las ciudades (Plaza, 1999, González, 2011). No obstante, el efecto de este nuevo modelo de políticas culturales sobre los grandes equipamientos culturales no ha recibido mucha atención.

En este artículo nos proponemos demostrar que los modelos de política cultural ya no están determinados por los modelos nacionales de política cultural tal y como han sido caracterizados a finales del siglo XX (Zimmer y Toepler, 1996, Mulcahy, 1998, Zimmer y Toepler, 1999) sino que hoy en día dependen en gran medida de los modelos locales de desarrollo de la política cultural. Estos nuevos modelos de política cultural de base local, que podemos calificar como híbridos, produce un nuevo modelo de gobernanza, titularidad y financiación de los equipamientos culturales que también podemos calificar de híbridos, ni públicos ni privados (Schuster, 1998) y en el que se mezclan los rasgos de los modelos europeocontinental y liberal de equipamientos culturales. Analizaremos también cómo este modelo, que también debe ser situado en el marco de una estrategia emprendedora a nivel local, tiene unos resultados positivos si se considera su capacidad para potenciar a corto y medio plazo la política cultural, pese a que también genera algunos problemas de gobernanza a largo plazo.

Para poder analizar el nuevo modelo nos centraremos en el caso de la ciudad de Barcelona que, como veremos, representa un caso avanzado de esta estrategia. Pero antes de entrar en el análisis de caso, en primer lugar, se abordará la definición de los modelos de políticas culturales y su correlación con unos modelos de gestión de 
los grandes equipamientos culturales ${ }^{1}$. En segundo lugar se definirá el Modelo Barcelona de desarrollo urbano y su influencia en el modelo de política cultural. Una influencia que se plasma en la utilización de los equipamientos culturales para la creación de clústeres culturales en el Raval, Glòries y Montjuïc. En tercer lugar, se analizarán los equipamientos culturales desde el punto de vista de la gobernanza, la financiación y la titularidad, destacando su carácter de modelo híbrido. A continuación, se analizará el desarrollo de cinco casos de equipamientos culturales caracterizados por ser el producto y ejemplo del modelo Barcelona de equipamientos culturales. Finalmente se llevará a cabo una valoración de las ventajas y los inconvenientes de este modelo de gestión local de las políticas y los equipamientos culturales.

Para la elaboración de este artículo, hemos utilizado las fuentes documentales como las memorias, los presupuestos y los documentos elaborados por la propia administración, así como el análisis de contenidos de las entrevistas a los responsables de la política cultural autonómica y local, y a los directores de las organizaciones culturales nacionales, generadas en el marco una investigación sobre el sistema de la política cultural en Cataluña ${ }^{2}$.

1 En este artículo se estudiarán los equipamientos culturales nacionales de Barcelona que generan actividad cultural de cara al público, reciben financiación pública para su funcionamiento ordinario y cuentan con autonomía jurídica y financiera. Con esta definición se engloban los museos, teatros y auditorios que superan el millón de euros de presupuesto y que se encuentran en la ciudad de Barcelona. Se excluyen los equipamientos patrimoniales que no ofrecen un servicio directo al ciudadano como la Biblioteca Nacional de Cataluña o el Archivo Nacional de Cataluña (ambos reservados a los investigadores), los teatros de titularidad y gestión privada y con afán de lucro y los equipamientos culturales con un presupuesto inferior al millón de euros. Entendemos que esta elección puede ser discutible, pero es necesaria para delimitar correctamente el objeto de estudio. Hemos excluido del análisis los equipamientos que son un servicio directo de las administraciones públicas, como es el caso del Museo Picasso de Barcelona, que no tiene personalidad jurídica ni presupuesto propio, a pesar de ser uno de los museos más visitados especialmente por los turistas que visitan la ciudad (Turisme de Barcelona, 2012).

${ }^{2}$ La investigación se ha desarrollado entre 2008 y 2011 y ha sido financiada por el Ministerio de Ciencia e Innovación (Ref. CSO2008-05910/SOCI). En el marco de este proyecto se ha entrevistado a responsables de la política cultural: Ferran Mascarell (Consejero de Cultura), Lluís Noguera (ex Secretario General del Departamento de Cultura), Oriol Picas (ex Jefe de la Oficina de Difusión Artística de la Diputación de Barcelona), Jordi Martí (ex Regidor de Cultura del Ayuntamiento de Barcelona), Jaume Ciurana (Regidor de Cultura y Educación del Ayuntamiento de Barcelona) y Marta Clari (Gerente del Área de Cultura del Ayuntamiento de Barcelona). También se ha entrevistado a una amplia muestra de los máximos responsables las principales instituciones culturales de Barcelona: Pepe Serra (Director del Museo Nacional de Arte de Catalunya), Bartomeu Marí (Director del Museo de Arte Contemporáneo de Barcelona), Sergi Belbel (Director del Teatro Nacional de Cataluña), Lluís Pasqual (Director del Teatro Lliure), Francesc Casadesús (Director del 


\section{Modelos de políticas culturales y tipos de equipamientos culturales: de los modelos nacionales a los modelos locales}

Los tipos de equipamientos culturales que podemos encontrar en cada país están determinados por el modelo de política cultural que se ha configurado a nivel histórico. El modelo de política cultural nacional tradicionalmente ha definido un contexto institucional que ha marcado durante la segunda mitad del siglo XX una pauta de relación entre las instituciones culturales y las administraciones públicas (Zimmer y Toepler, 1999). A partir de los años ochenta y especialmente durante los años noventa, el desarrollo de modelos locales de política cultural y el rol de instrumental jugado por las instituciones culturales han condicionado de forma creciente las instituciones culturales. Examinaremos, por lo tanto, a continuación la influencia de los modelos estatales y posteriormente la de los modelos locales.

\subsection{El modelo liberal y centro-europeo de política cultural y tipos ideales de instituciones culturales}

En sus estudios comparativos sobre los sistemas de política cultural que se implantan en los diferentes países occidentales tradicionalmente se han distinguido tres modelos de políticas culturales (Zimmer y Toepler, 1996). Siguiendo el análisis de los tres modelos de estado de bienestar de Esping-Andersen (1993) podemos establecer tres modelos: el europeo-continental, el anglosajón y el nórdico. En este artículo analizaremos los dos primeros, por ser los relevantes para el caso barcelonés.

En el modelo europeo-continental, de raíz absolutista, existe una red de instituciones artísticas orientadas a la excelencia que, al mismo tiempo, cuentan con una fuerte tradición de intervención pública. El gobierno central en este modelo posee el rol predominante y ejerce una función de dominio político y simbólico respecto de los otros niveles administrativos. El papel del sector privado en este caso es subsidiario y existen pocos casos de cooperación público-privada. El caso paradigmático de este modelo es el de Francia (Urfalino, 1996).

Por el contrario, en el modelo liberal, en ausencia de una tradición absolutista, el apoyo tradicional de las instituciones culturales ha estado en manos privadas. El mercado constituye en estos países el mecanismo de regulación de la vida cultural por excelencia y la intervención del estado es menor, como es el caso de Gran Bretaña (Moulin, 1992).

Centro de danza Mercat de les Flors), Joaquim Garrigosa (Director del Auditorio de Barcelona), Joan Oller (Director del Palau de la Música), Rosa Maria Malet (Directora de la Fundación Joan Miró) y Laurence Rassel (Directora de la Fundación Antoni Tàpies). 
Aunque no podemos establecer una correlación perfecta entre el modelo de política cultural y el modelo de equipamiento cultural ${ }^{3}$, siguiendo los modelos de política cultural planteados anteriormente (Zimmer y Toepler, 1996), podemos construir un tipo ideal de equipamientos culturales de tipo anglosajón, europeo-continental según el modelo de política cultural (ver Tabla 1).

Tabla 1: Modelos de política cultural y tipo ideal de equipamiento cultural

\begin{tabular}{|c|c|c|}
\hline Titularidad edificio & $\begin{array}{c}\text { Modelo europeo- } \\
\text { continental }\end{array}$ & Modelo anglosajón \\
\hline Titularidad gestión & $\begin{array}{c}\text { Gestión directa u } \\
\text { organismo autónomo }\end{array}$ & $\begin{array}{c}\text { Organización sin } \\
\text { ánimo de lucro }\end{array}$ \\
\hline Titularidad colección & Sector público & Tercer sector \\
\hline Patronato & $\begin{array}{c}\text { Representantes } \\
\text { instituciones }\end{array}$ & $\begin{array}{c}\text { Patronos independien- } \\
\text { tes }\end{array}$ \\
\hline
\end{tabular}

Fuente: elaboración propia a partir de Zimmer y Toepler (1996) y de Lord y Lord (1998)

Como se puede ver en la Tabla 1, el modelo predominante de los equipamientos culturales en el contexto de políticas culturales europeo-continentales es el de titularidad pública con gestión directa o, en todo caso, a partir de organismos dependientes de las administraciones públicas. Fuertemente subvencionados, su gestión está controlada directamente por los representantes públicos que, entre otras facultades, nombran al director. Si bien se ejerce un claro control ex ante por parte del Ministerio de Economía, la dirección de la actividad del equipamiento es potestad del director artístico y no se suelen establecer objetivos de gestión e impacto social. Este suele ser el modelo de las instituciones culturales de países europeocontinentales (Coppinger et al., 2007) aunque también el modelo de las instituciones culturales en algunos países mediterráneos como Italia (Fedeli y Santoni, 2006).

3 Existe una abundante literatura que analiza desde el punto de vista institucional y económico las diferentes fórmulas de gestión según su proximidad al sector público o al sector privado. Por ejemplo, Lord y Lord (Lord y Lord, 1998) analizan cuatro modelos diferentes: dependencia orgánica de la administración pública, dependencia de la administración pública con autonomía de gestión, organización sin fines de lucro o entidad privada. En el caso de España, no existen grandes equipamientos culturales estrictamente privados, es decir, pertenecientes al sector privado lucrativo, sino que todos pueden ser considerados del sector privado no lucrativo o Tercer sector. 
En el modelo anglosajón, por el contrario, nos encontraríamos con equipamientos culturales de titularidad privada (mayoritariamente en el sector no lucrativo), con órganos de gestión autónomos respecto de las administraciones públicas. Su equipo directivo, apoyado por un patronato capacitado e independiente, conseguiría más ingresos propios provenientes de la taquilla, el mecenazgo y el patrocinio. Por lo tanto, la titularidad y la gestión de este tipo de equipamientos es privado nonprofit, aunque muchos de ellos, como es el caso de Gran Bretaña, reciben fondos del gobierno o de sus agencias como los consejos de las artes (Department of Culture, Media and Sport, 2010).

Desde los años ochenta, se ha apreciado una tendencia a la disminución de las diferencias entre estos dos modelos con un incremento de la intervención del estado en los países del modelo liberal, y un mayor rol del sector privado y el tercer sector en los países del modelo europeo-continental (Bianchini, 1993a). La disminución en las subvenciones públicas (Dewey, 2004), la influencia de la Nueva Gestión Pública y sus reformas desestatizadoras (Belfiore, 2004), la globalización cultural así como la mayor importancia de las industrias culturales y creativas explican, en parte, esta convergencia (Menger, 2010, DEPS, 2011).

\section{La emergencia de modelos locales de política cultural y los nuevos tipos de equipamientos culturales}

En el contexto del paso a la economía fordista y de la aparición de los paradigmas de la ciudad creativa (Landry y Bianchini, 1995) el marco local ha tomado un nuevo protagonismo y sus gobiernos han cambiado y han ganado peso. Así, han pasado de ser pasivos implementadores de las políticas estatales de servicios públicos a ser activos promotores del desarrollo local (I. Blanco, 2009a). Tanto el llamado entrepreneurial turn (Harvey, 1989) de las políticas locales que apuesta por la revitalización urbana sobre la base de grandes proyectos arquitectónicos y eventos espectaculares, como el desarrollo de servicios y de nuevas industrias, tienen un eminente carácter cultural, que a menudo se concreta en la creación de barrios artísticos o de clústeres de industrias culturales (Scott, 2000, Scott, 2010). Así, se afirma que las estrategias culturales se tornan claves para la supervivencia de las ciudades (Zukin, 1995: 271). Uno de los mecanismos de extensión de la utilización de los grandes eventos como catalizadores del desarrollo urbano ha sido la construcción de museos bandera (Bianchini, 1993b) o la generación de grandes eventos (García, 2004). A partir de estas actuaciones se gestó un nuevo modelo de política cultural, que, como en el caso de Liverpool y Barcelona, representan la voluntad de unir cambio urbano, desarrollo económico y transformación social (Connolly, 2011). Así, a partir de los años ochenta la política cultural es concebida como un motor de la economía de las ciudades y una palanca de la regeneración de los centros urbanos (Landry y Bianchini, 1995).

Asimismo, se inicia entonces una tendencia a instrumentalizar la política cultural para fines de otras agendas de política pública (Gray, 2007), a pesar de que no se ha 
demostrado de forma empírica su efectividad (Belfiore, 2002). En este contexto se produce un cambio en los objetivos de la política cultural como política social, respaldado por un giro conceptual en el que la concepción redistributiva típica de las políticas culturales estatales (Menger, 2010) es desplazada por la idea de la cultura como factor de inclusión social (Connolly, 2011).

Esta nueva política cultural se está concibiendo cada vez más desde una perspectiva sistémica y estratégica (Cherbo y Wyszomirski, 2000) y es en las ciudades donde esta nueva sistematicidad se está fraguando de un modo más tangible (Evans, 2001: 221), que se expresa en nuevas formas de planificación y gobernanza cultural y orienta la política cultural hacia una estrategia de fertilización intersectorial con el objetivo de crear valor cultural local (Currid, 2007) en un contexto de competitividad global entre ciudades creativas (Menger, 2010). En este marco se transforma la dimensión organizativa de la política cultural bajo los principios de la Nueva Gestión Pública (Belfiore, 2004), agencializando e instrumentalizando las organizaciones culturales financiadas públicamente para la consecución de estos nuevos objetivos (Gray, 2008, J. Rius y Rubio, 2013).

Podemos por lo tanto hablar del surgimiento de nuevos modelos híbridos de política cultural, protagonizados especialmente por gobiernos regionales y locales (Gattinger y Saint-Pierre, 2008). Este artículo mostrará que estos nuevos modelos locales están generando lo que Mark Schuster calificó como equipamientos culturales híbridos (Schuster, 1998), es decir, ni públicos ni privados, y que no se corresponden ni con el modelo europeo-continental ni con el liberal. Por otra parte, la instrumentalización de la política cultural local comportará también que los grandes equipamientos sean concebidos como instrumentos para la regeneración urbanística, la promoción económica y la cohesión social. No obstante, la forma en la que ello se expresa en cada ciudad dependerá de los modelos locales de política cultural local, que son combinaciones diferenciadas de los retos y dilemas de la política cultural urbana planteados hace ya veinte años (Bianchini, 1993a) y que siguen vigentes: efecto final vs. valor cultural, grandes eventos vs. pequeñas acciones, proyección internacional vs. desarrollo local. Dilemas que el Modelo Barcelona ha intentado -con éxito desigual- resolver mediante un modelo propio de desarrollo urbano y política cultural. A continuación, analizaremos cómo se ha generado un modelo propio de política cultural local en Barcelona, coherente con el llamado Modelo Barcelona de desarrollo urbano, que ha producido un modelo específico de equipamiento cultural.

\subsection{El Modelo Barcelona: transformación urbana y política cultural}

El Modelo Barcelona se entiende como un proyecto de ciudad liderado por el gobierno local, con el objetivo de transformar económica, social y culturalmente la 
ciudad. Asimismo, se ha analizado como un impulso modernizador de la ciudad bajo los sucesivos gobiernos de orientación socialdemócrata ${ }^{4}$, caracterizado por la aplicación de un urbanismo socialmente inclusivo y culturalmente avanzado (Marshall, 2000). Entre los elementos habitualmente atribuidos a este modelo podemos destacar los siguientes elementos: a) el liderazgo del gobierno local con una agenda que pretende combinar de forma equilibrada transformación urbana, desarrollo económico y cohesión social; b) la utilización de grandes eventos y de la cultura como estrategias de transformación simbólico-material de la ciudad (Subirós, 1998): c) la introducción de la lógica de la planificación estratégica y de herramientas de la Nueva Gestión Pública; y d) La gobernanza o la cooperación entre distintos niveles de gobierno y el partenariado público-privado en la generación de proyectos urbanos de interés público (I. Blanco, 2009b).

Tal como señala Arturo Rodríguez (2005, 2008) la estrategia cultural barcelonesa deriva de una larga trayectoria hasta llegar a nuestros días. Durante la segunda mitad del siglo XIX y durante el primer tercio del siglo XX, la acción concertada de las elites locales y del poder local crea instituciones culturales de prestigio y promueve grandes eventos, como las exposiciones universales de 1888 y 1929. Esta singular tradición de activismo mancomunado de las elites y el poder local resurge en los años ochenta, época en la que Barcelona desarrollará uno de los modelos más avanzados de ciudad emprendedora (Marshall, 1996).

Este modelo de ciudad emprendedora en el desarrollo local se ha reflejado en la importancia otorgada a la planificación estratégica local, de la que el Ayuntamiento de Barcelona ha sido pionero, y con la que se impulsará el nacimiento de un modelo de políticas culturales barcelonés (Guillemat, 2008). Se partía de la idea de fomentar la capacidad de crear valor cultural local a través del fortalecimiento de los vínculos entre todos los actores que intervienen en el ámbito de la cultura (públicoprivado-tercer sector), y en todas las fases de la cadena de producción cultural (creación-producción-difusión), así como de promover colaboraciones interdisciplinares. A medio camino entre dispositivo de planificación, de participación y de gobernanza, los planes estratégicos han mostrado la creciente consciencia del sector cultural por las interrelaciones entre los diversos sectores y segmentos, así como su rol central en el desarrollo social y económico de la ciudad. Sin embargo, este proceso de reconocimiento y legitimación de la cultura como recurso para el desarrollo social y económico, no tuvo la misma intensidad en relación con su potencial uso como herramienta y como espacio para la participación de la ciudadanía y de los profesionales del sector cultural.

${ }^{4}$ Las primeras elecciones municipales democráticas de 1979 dieron la victoria al Partido de los Socialistas de Cataluña que desde entonces gobernó la ciudad de forma ininterrumpida hasta que en 2011 Convergencia i Unió, una coalición de la derecha nacionalista, consiguió su primera victoria electoral en la capital catalana. 
El primer plan se elabora en 1999 y marca un hito en esta nueva etapa en la que la cultura se conceptualiza como un capital que debe movilizarse conjuntamente entre el sector público y el privado para convertir Barcelona en una centro productor de cultura (ICUB, 1999). En este contexto se pretende convertir los equipamientos culturales en factorías de creación de contenidos a partir de acoger en su seno plataformas de creación emergente (ídem: 5).

El Plan del 2006, llamado "Nuevos acentos", partía de la constatación de que los objetivos del Plan Estratégico de 1999 -posicionar la cultura como un recurso clave en el marco de la estrategia de la ciudad, así como reconocer el vínculo entre cultura y desarrollo económico y tecnológico- se habían conseguido en su mayor parte. No obstante, el nuevo plan perseguía "volver a poner la cultura como finalidad de las políticas culturales” (ICUB, 2006). Ello suponía, por lo tanto, sin negar los efectos beneficiosos en lo económico y en lo social, evitar los desbordamientos instrumentalizadores y situar en el centro la potenciación de los sectores culturales (Guillemat, 2008). Para ayudar a esta capacidad de creación de valor cultural se concebía Barcelona como un laboratorio al que había que facilitar la conectividad y la participación. Por ello dos de los programas estrella fueron la creación de las Fábricas de la Creación, que volvieron a concebir los equipamientos culturales como un elemento clave en el intento de convertir la política cultural en una herramienta de desarrollo de un sector cultural capaz de generar valor cultural para proyectarlo a nivel regional, nacional e internacional.

Existe cierto consenso en que el Modelo Barcelona es un modelo de éxito en el que se combinó de forma acertada renovación urbana y planificación cultural (Subirós, 1999). No obstante, desde su formulación como modelo, diversos autores han desarrollado diferentes críticas, a saber, su deriva de modelo a marca de ciudad mercantilizada (Balibrea, 2001), su instrumentalización neoliberal y autoritaria de la cultura (Degen y García, 2012) que se ha constatado con fuerza en el último gran evento de la ciudad, El Fórum Universal de las Culturas 2004 (Majoor, 2011).

En correspondencia con lo que hemos argumentado podemos establecer la existencia de un Modelo Barcelona de políticas culturales que se caracteriza por: a) un liderazgo de la administración local muy por encima de la definición competencial en cultura con la intención de convertir la cultura en un sector estratégico y que se evidencia a nivel organizativo, presupuestario y en la gobernanza de las instituciones culturales; b) la utilización de la regeneración urbana y de los grandes eventos como oportunidad de desarrollo de las instituciones culturales; c) la creciente importancia de la cultura dentro de la planificación estratégica local y la elaboración de contratos programa de los equipamientos culturales como factor de instrumentalización de las políticas culturales y d) la potenciación de la cooperación públicoprivada como oportunidad de desarrollo de los sectores culturales en su conjunto. 


\section{Modelo de política cultural y modelo de equipamientos culturales: el caso de Barcelona}

\subsection{Génesis de los equipamientos culturales de Barcelona y liderazgo empren- dedor local}

En el Estado español no existe un modelo único de políticas culturales y de gobernanza y gestión de los equipamientos culturales. De hecho, podemos encontrar modelos diferentes en su territorio. Por una parte, el caso de Madrid sería asimilable al modelo europeo-continental (Rubio Arostegui, 2008, Rubio y Rius, 2012). Por el contrario, Barcelona ha estado históricamente cercana al modelo liberal (Rodriguez Morató, 2008). La debilidad de la intervención del Estado en la esfera cultural durante el siglo XIX y buena parte del siglo XX, comportó que los equipamientos culturales estuvieran mayoritariamente en manos de la iniciativa privada o del tercer sector.

Este es el caso, por ejemplo, del Gran Teatre del Liceu ${ }^{5}$ (GTL), la mayor institución cultural barcelonesa y un referente a nivel local e internacional de la escena operística y de la imagen de Barcelona como ciudad cultural. Aunque actualmente se considera una de las piezas esenciales de la política cultural barcelonesa, el Liceu fue en su origen y durante un siglo y medio una entidad privada. De hecho, el Liceu fue creado en 1847 por iniciativa de miembros destacados de la burguesía local y la explotación del teatro fue encomendada a diversas empresas concesionarias. Este modelo de gestión perduró hasta finales del siglo XX, un momento en el que los grandes teatros de ópera de los países avanzados ya eran o bien públicos, o bien recibían cuantiosas subvenciones del Estado (Martorella, 1982). En los años setenta El Liceu entró en una crisis de público y de modelo de gestión (Alier, 1978), una situación está que amenazó la continuidad del teatro y que forzó a las administraciones públicas a intervenir y a crear en 1980 el Consorcio del Gran Teatre del Liceu, integrado por el Departamento de Cultura, el Ayuntamiento de Barcelona y la Societat del Gran Teatre del Liceu, que detentaría la titularidad del inmueble hasta 1994. Posteriormente, se sumaron la Diputación de Barcelona en 1985 y el Ministerio de Cultura en 1986.

El 1994 un incendio destruyó completamente el auditorio, con la excepción de la fachada y de las salas anexas. Inmediatamente se generó un fuerte consenso entre las elites económicas, culturales y políticas sobre la necesidad de reconstruirlo rápidamente, que se reflejó en la constitución de la nueva Fundación Gran Teatre

${ }^{5}$ En este artículo hemos optado por mantener el nombre en catalán de las instituciones culturales barcelonesas. En algunos casos, hemos utilizado abreviaturas utilizadas de forma común en el sector cultural: el Liceu para denominar el Gran Teatre del Liceu, l'Auditori para denominar el Auditori de Barcelona y el Lliure para denominar el Teatre Lliure. 
del Liceu, integrada por las cuatro administraciones públicas mencionadas anteriormente, que aparcaron en este caso sus importantes diferencias sobre las competencias en cultura (Font, 1991). Este consenso se escenificó bajo el lema "El Liceu de todos" en oposición al Liceu de las elites que había representado hasta entonces, legitimando de este modo la fuerte inversión pública que había supuesto su reconstrucción (Caminal, 2004).

También el Palau de la Música Catalana, una de las joyas de la corona de los equipamientos culturales de Barcelona, debe su origen a la iniciativa local privada. Su edificio de estilo modernista fue construido a principios de siglo XX por el arquitecto Lluís Domènech i Montaner como sede de la asociación coral Orfeó Català y fue sufragado por la sociedad civil barcelonesa. Durante todo el siglo XX ha sido uno de los principales referentes culturales y una de las señas de identidad de Catalunya y de Barcelona.

Su proceso de integración en el sector público ha sido paulatino y no carente de dificultades y momentos de crisis. El primer paso de acercamiento a las administraciones públicas se produjo en los inicios de la recuperación de la democracia cuando la Junta Directiva del Palau solicitó ayuda para mantener el histórico edificio. Para vehicular las ayudas se constituyó el Consorcio en 1983, integrado por el Ministerio de Cultura, el Departamento de Cultura y el Ayuntamiento de Barcelona. Sin embargo, a cambio de la importante inversión pública no se transformó el estatuto jurídico del edificio del Palau que continuó siendo propiedad del Orfeó Català. En 1990, para gestionar la actividad del Palau se constituyó una fundación privada con mayoría de patronos privados pero con la participación de representantes de las mismas administraciones públicas presentes en el Consorcio.

Además de las instituciones históricas, en Barcelona podemos encontrar museos y centros de arte fruto de la iniciativa de la sociedad civil con la colaboración del Ayuntamiento de Barcelona en sus inicios y el resto de las administraciones públicas después. La Fundació Miró es una fundación privada con la misión de dar a conocer la obra del artista Joan Miró y de promover el arte contemporáneo. Fue fundada el año 1975 por iniciativa de intelectuales, artistas, marchantes y amigos del artista, y fue apoyada por el gobierno local desde sus inicios. Es una de las grandes instituciones culturales que más se aproximaría al modelo liberal, pero recibe anualmente aportaciones públicas y los representantes de las administraciones públicas están presentes en su patronato.

La Fundació Antoni Tàpies fue creada 1984 por el propio artista catalán, Antoni Tàpies, con el objetivo de promover el estudio y el conocimiento del arte contemporáneo, así como la difusión de la obra del propio artista. Aunque es una fundación privada, abrió sus puertas en 1990 en la sede de la antigua Editorial Montaner y Simon, propiedad del Ayuntamiento de Barcelona, y durante estos veinte años de actividad, las aportaciones de las administraciones han ido creciendo hasta llegar a suponer, en la actualidad, casi la mitad de los ingresos.

También fue iniciativa del sector privado el Teatre Lliure. El origen de esta institución cultural se remonta al período de la transición a la democracia, en 1976, cuando una compañía de teatro se constituyó en cooperativa. Desde entonces hasta 
1987, el Teatre Lliure se convirtió en uno de los referentes del teatro independiente, recibiendo por ello el apoyo del Ayuntamiento de Barcelona. En 1988 se constituyó la Fundación privada Teatre Lliure-Teatre Públic de Barcelona por parte de los representantes de la cooperativa de actores con la intención de conservar la independencia del teatro y así recibir un mayor apoyo de las administraciones públicas. Un apoyo imprescindible para poder financiar un nuevo espacio escénico que cumpliera con las necesidades técnicas y de seguridad que el antiguo espacio de Gracia ya no garantizaba. Así pues, con la complicidad del Ayuntamiento de Barcelona, que quería dar continuidad a una de las instituciones teatrales con mayor prestigio y dinamismo de la ciudad, se consiguió incorporar al Patronato, tanto a la Diputación de Barcelona, como a la Generalitat de Catalunya, así como al Ministerio de Cultura y su apoyo para construir la nueva sede donde se encuentra actualmente, en Montjuïc.

No es hasta la transición a la democracia cuando la nueva administración pública cultural autonómica, el Departamento de Cultura de la Generalitat de Catalunya ${ }^{6}$, empieza a intervenir en la cultura catalana y barcelonesa con el objetivo de modernizar las infraestructuras culturales y equipararlas a los estándares culturales europeos. Esta intención se plasmará en la construcción de nuevos equipamientos culturales como el Teatre Nacional de Catalunya (TNC) en 1997, inspirado en el modelo francés de institución democratizadora de la alta cultura. También fue fruto de la voluntad de dotarse de instituciones nacionales la construcción, a finales de los años noventa, del Auditori de Barcelona, que convertía la orquestra de titularidad local en nacional y, el Museu Nacional d'Art de Catalunya, que se convertiría en el escaparate del arte catalán. También el Mercat de les Flors ha respondido de la misma manera a esta intención del gobierno local de dotar a cada sector cultural, en este caso la danza, de una gran institución cultural de referencia.

No obstante, este no fue el paradigma de actuación dominante y, en realidad, durante los años ochenta y noventa la política cultural y el desarrollo de equipamientos culturales en Cataluña estará liderado por los gobiernos locales. Estos, en ausencia de una planificación y un apoyo financiero por parte del Ministerio de Cultura o del Departamento de Cultura, desarrollarán estrategias emprendedoras para aprovechar las fortalezas y oportunidades locales (Martinez y Font, 2010).

Este fue el caso de la que podemos llamar "generación olímpica de equipamientos culturales, es decir, aquellos que se gestaron durante el periodo posterior a la nominación olímpica hasta mediados de los años noventa. En el contexto de la movilización de recursos públicos y privados que requirió ese gran evento deportivo, se proyectaron dos equipamientos culturales, el Museo de Arte Contemporáneo de Barcelona y el Centro de Cultura Contemporánea de Barcelona que se inauguraron

${ }^{6}$ Por Departamento de Cultura estamos refiriéndonos al organismo responsable de ejecutar las políticas culturales de la Generalitat de Catalunya. 
a mediados de los años noventa. Estos fueron utilizados como emblema de la regeneración urbana del centro de la ciudad, tal y como veremos a continuación.

\subsection{Desarrollo urbano, política cultural e instituciones culturales en Barcelo- na}

Una de las características del Modelo Barcelona de desarrollo urbano es la utilización de las grandes infraestructuras culturales para la regeneración urbana y la creación de nuevas centralidades ciudadanas. Esta voluntad se plasma en el modelo de política cultural que concibe a la vez los procesos de regeneración urbana como una oportunidad para conseguir la financiación para equipamientos culturales y generar unos clústeres culturales que consigan fraguar sinergias entre los propios equipamientos y atraer al público al generar una escena artística. Esta ha sido la voluntad en el caso de los clústeres del Raval, Glòries y Montjuïc.

Mapa 1: Clústeres de instituciones culturales de Barcelona

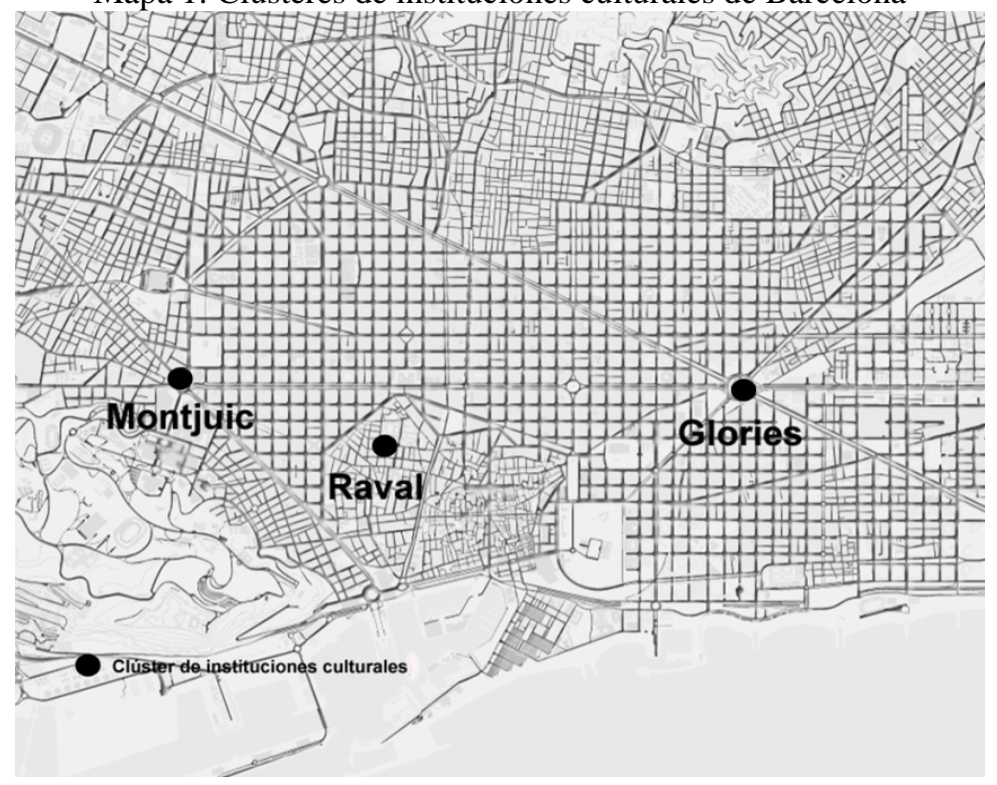

Fuente: elaboración propia

La idea de un clúster cultural en el Raval comienza a principios de los años ochenta con el motivo de dedicar a la cultura un espacio infrautilizado y en proceso de degradación en el norte del barrio. Entonces, la Diputación de Barcelona (DIBA) y el Ayuntamiento de Barcelona impulsan la creación de una gran institución cultural que federe diversos servicios culturales, siguiendo el modelo del Pompidou parisino. 
Los responsables de estas dos administraciones públicas entienden que las infraestructuras culturales ayudan al impulso de un proceso de regeneración urbana que se complementará con una fuerte inversión urbanística pública y privada (Subirats y Rius, 2008). El proyecto fundador, denominado Centre de Cultura de la Casa de la Caritat, se dividirá a mediados de los años ochenta en dos proyectos separados: el Centre de Cultura Contemporània de Barcelona (CCCB) y el Museu d'Art Contemporani de Barcelona (MACBA).

El clúster de instituciones culturales de Glòries nace a principios de la década del noventa cuando el entonces alcalde de Barcelona, Pascual Maragall, convence a los responsables de la Generalitat de Catalunya de situar su proyecto estrella, el Teatro Nacional de Catalunya (TNC), como una apuesta estratégica urbana (Maragall, 1989). Siguiendo esta lógica, al edificio del TNC, inaugurado en 1997, se le une dos años después el Auditorio de Música y en 2003 la Escuela Superior de Música de Catalunya en un edificio adyacente. Finalmente, se planifica para 2012 la inauguración del Disseny Hub de Barcelona (DHUB) en la misma zona para crear así una nueva centralidad urbana en esa zona de la ciudad.

El proyecto de la Ciutat del Teatre (ciudad del teatro) de Monjuïc empieza en 1997 con el encargo por parte del Ayuntamiento de Barcelona al prestigioso director teatral Lluís Pascual de liderar un proyecto que englobe diversas instituciones existentes o en construcción alrededor de la zona de Monjuïc (Pascual 2001). El proyecto coordina tres espacios teatrales distintos: el Mercat de les Flors (danza contemporánea), el nuevo Teatre Lliure (teatro) y el Instituto del Teatro (institución de enseñanza superior de teatro y danza). El proyecto liderado por el gobierno local tiene por finalidad la creación de sinergias culturales y un impacto urbano, y se suma al polo de museos formado por el Museu Nacional d'Art de Catalunya y la Fundació Joan Miró.

\subsection{Gobernanza y financiación pública multinivel de los grandes equipamien- tos culturales de Barcelona}

La convivencia de la herencia histórica surgida de la iniciativa privada, el modelo europeo-continental de equipamiento cultural desarrollado por el Departamento de Cultura y el modelo emprendedor impulsado por el Ayuntamiento de Barcelona han creado una tipología de equipamiento caracterizado por una gran complejidad en su estructura de gobernanza y fuentes de financiación.

Una de las características del modelo barcelonés es la extrema complejidad en la gobernanza de sus equipamientos culturales, dado que no hay una primacía de los niveles superiores de las administraciones públicas (Ministerio de Cultura -MC- y Departamento de Cultura -DC-), sino que el nivel local (Instituto de Cultura ICUB- y Diputación de Barcelona -DIBA) detentan un rol equivalente al de las otras administraciones, situación impensable en el Modelo centro-europeo o en el modelo liberal, en el que el Estado central tiene un protagonismo mayor. Por otra parte, solamente dos dependen de una sola administración: el Museu Picasso y el 
Teatre Nacional de Catalunya. El Museu Picasso porque se ha convertido en símbolo de la política cultural barcelonesa y de su proyección internacional, y el Ayuntamiento recela de compartirlo con las otras administraciones. Y el TNC, porque fue construido como el símbolo de la actuación del nuevo Departamento de Cultura del gobierno autonómico y su estrategia de reivindicación nacionalista (Villarroya, 2012). El resto de equipamientos culturales dependen de dos administraciones públicas (Centre de Cultura Contemporània de Barcelona, Fundació Antoni Tàpies, MACBA, Mercat de les Flors), de tres (Auditori, Fundació Joan Miró, MNAC) y hasta de cuatro (Liceu, Palau de la Música y Teatre Lliure).

Tabla 2: Grandes equipamientos culturales y número de administraciones públicas titulares (2012)

\begin{tabular}{|l|c|c|c|c|c|}
\hline & ICUB & DIBA & DC & MC & TOTAL \\
\hline Auditori (AUD) & 1 & & 1 & 1 & $\mathbf{3}$ \\
\hline $\begin{array}{l}\text { Centre de Cultura Contemporània de Barcelona } \\
\text { (CCCB) }\end{array}$ & 1 & 1 & & & $\mathbf{2}$ \\
\hline Fundació Antoni Tàpies (FAT) & 1 & & 1 & & $\mathbf{2}$ \\
\hline Fundació Joan Miró (FJM) & 1 & & 1 & 1 & $\mathbf{3}$ \\
\hline Gran Teatre del Liceu (GTL) & 1 & 1 & 1 & 1 & $\mathbf{4}$ \\
\hline $\begin{array}{l}\text { Museu d'Art Contemporani de Barcelona } \\
\text { (MACBA) }\end{array}$ & 1 & & & & $\mathbf{2}$ \\
\hline Mercat de les Flors (MF) & 1 & & 1 & & $\mathbf{2}$ \\
\hline Museu Nacional d'Art de Catalunya (MNAC) & 1 & & 1 & 1 & $\mathbf{3}$ \\
\hline Museu Picasso de Barcelona (MPB) & 1 & & & & $\mathbf{1}$ \\
\hline Palau de la Música (PM) & 1 & 1 & 1 & 1 & $\mathbf{4}$ \\
\hline Teatre Lliure (TLL) & 1 & 1 & 1 & 1 & $\mathbf{4}$ \\
\hline Teatre Nacional de Catalunya (TNC) & & & 1 & & $\mathbf{1}$ \\
\hline TOTAL & $\mathbf{1 1}$ & $\mathbf{4}$ & $\mathbf{1 0}$ & $\mathbf{6}$ & \\
\hline
\end{tabular}

Fuente: elaboración propia

En cuanto a la participación de las administraciones culturales, podemos comprobar que el Departamento de Cultura (DC), que es la administración con competencias exclusivas sobre cultura, solo está presente en los diez equipamientos culturales, viéndose superado por el gobierno local que forma parte de las estructuras de gobernanza de once equipamientos culturales a través de su instituto autónomo de cultura, el Instituto de Cultura de Barcelona. Por su parte el Ministerio de Cultura

${ }^{7}$ Por ICUB nos referimos al Instituto de Cultura de Barcelona del Ayuntamiento de Barcelona, por DIBA nos referimos al Área de Cultura de la Diputación de Barcelona, por DC nos referimos al Departamento de Cultura de la Generalitat de Catalunya y por MC nos referimos a Ministerio de Cultura de la Administración General del Estado. 
tiene una presencia mucho menor, al estar presente solamente en seis de los diez grandes equipamientos culturales.

Tabla 3: Financiación de los equipamientos culturales de Barcelona por administración pública (temporada 2009-2010). Miles de euros.

\begin{tabular}{|c|c|c|c|c|c|c|c|c|c|c|}
\hline & DC & $\%$ & ICUB & $\%$ & DIBA & $\%$ & MC & $\%$ & TOTAL & $\%$ \\
\hline Auditori (AUD) & 8.357 & 49 & 7.615 & 45 & & 0 & 950 & 6 & 16.922 & 100 \\
\hline $\begin{array}{l}\text { Centre de Cultura } \\
\text { Contemporània de } \\
\text { Barcelona (CCCB) }\end{array}$ & & 0 & 2.657 & 25 & 7.973 & 75 & & & 10.630 & 100 \\
\hline $\begin{array}{l}\text { Fundació Antoni } \\
\text { Tàpies (FAT) }\end{array}$ & 365 & 43 & 391 & 46 & & 0 & 95 & 11 & 851 & 100 \\
\hline $\begin{array}{l}\text { Fundació Joan Miró } \\
\text { (FJM) }\end{array}$ & 827 & 43 & 903 & 47 & & 0 & 190 & 10 & 1.920 & 100 \\
\hline $\begin{array}{l}\text { Gran Teatre del } \\
\text { Liceu (GTL) }\end{array}$ & 10.682 & 38 & 2.961 & 10 & 1.480 & 5 & 13.326 & 47 & 28.449 & 100 \\
\hline $\begin{array}{l}\text { Museu d'Art } \\
\text { Contemporani de } \\
\text { Barcelona } \\
\text { (MACBA) } \\
\end{array}$ & 4.516 & 40 & 4.766 & 42 & & 0 & 2.000 & 18 & 11.282 & 100 \\
\hline $\begin{array}{l}\text { Mercat de les Flors } \\
\text { (MF) }\end{array}$ & 2.484 & 40 & 2.461 & 40 & & 0 & 1.235 & 20 & 6.180 & 100 \\
\hline $\begin{array}{l}\text { Museu Nacional } \\
\text { d'Art de Catalunya } \\
\text { (MNAC) }\end{array}$ & 9.461 & 47 & 5.016 & 25 & & 0 & 5.817 & 29 & 20.294 & 100 \\
\hline $\begin{array}{l}\text { Museu Picasso de } \\
\text { Barcelona (MPB) }\end{array}$ & & 0 & 4.200 & 100 & & 0 & & 0 & 4.200 & 100 \\
\hline $\begin{array}{l}\text { Palau de la Música } \\
\text { (PM) }\end{array}$ & 1.734 & 55 & 749 & 24 & 55 & 2 & 588 & 19 & 3.126 & 100 \\
\hline Teatre Lliure (TLL) & 2.499 & 38 & 2.732 & 42 & 444 & 7 & 900 & 14 & 6.575 & 100 \\
\hline $\begin{array}{l}\text { Teatre Nacional de } \\
\text { Catalunya (TNC) }\end{array}$ & 11.541 & 100 & & 0 & & 0 & & 0 & 11.541 & 100 \\
\hline TOTAL & 52.466 & 43 & 34.451 & 28 & 9.952 & 8 & 25.101 & 21 & 121.970 & 100 \\
\hline
\end{tabular}

Fuente: elaboración propia a partir de los datos presupuestarios 2009-2010

${ }^{8}$ Las fuentes de los datos presupuestarios utilizados son las siguientes: memorias presupuestarias de la temporada 2009-2010 disponibles en los archivos del Departamento de Cultura, los presupuestos de la Generalitat de Catalunya (Generalitat de Catalunya, 2010), los presupuestos del Ministerio de Cultura (Ministerio de Economía y Hacienda, 2009) y la Memoria del Instituto de Cultura de Barcelona (Generalitat de Catalunya, 2010). Se han excluido del análisis las subvenciones a actividades concretas desarrolladas por los equipamientos culturales (exposiciones, conciertos u otras actividades culturales) porque se trata de partidas de pocos miles de euros, son muy variables según el año, no se conceden regularmente y no son informativas, por lo tanto, de la estructura de financiación de las organizaciones culturales nacionales. 
Como podemos ver en la Tabla 3, el DC asume casi la mitad de las transferencias públicas a los equipamientos culturales, y en una segunda posición aparece el Ayuntamiento de Barcelona con una contribución del 28\% del total, muy por encima de sus responsabilidades como administración local, seguido en un tercer lugar por el Ministerio de Cultura que solamente asume 25 millones de euros (un 21\% del total), muy por debajo de los 179 millones que dedica a los equipamientos culturales de Madrid.

Así pues, el repartimiento de las responsabilidades de gobernanza y de la financiación entre las diferentes administraciones públicas nos indica que Barcelona sigue estando muy lejos del modelo europeo-continental en el que el Ministerio de Cultura central tiene una participación mayoritaria en todos los grandes equipamientos culturales, tanto en su gobernanza como en su financiación. En este caso, los gobiernos autonómico y local tienen una participación muy superior a la que se esperaría en este tipo de equipamientos culturales de gran dimensión que responde, como hemos mencionado, a esta orientación emprendedora de la política cultural local que ha consistido en buscar la complicidad de todos los niveles de las administraciones públicas para desarrollar unos equipamientos culturales sin cuya participación no hubieran sido financieramente posibles.

\subsection{La cooperación público-privada en los equipamientos cultuales de Barce- lona}

En primer lugar, podemos constatar que de las doce instituciones culturales analizadas, cuatro corresponderían de forma clara al sector público porque son el resultado de una iniciativa de las administraciones públicas hasta el punto de que la titularidad del edificio, la gestión y la colección son públicas (Auditori, Mercat de les Flors, MNAC y TNC). Sin embargo el resto de instituciones culturales, al combinar titularidades públicas y privadas deben ser calificadas como organizaciones híbridas. Es el caso de la Fundació Antoni Tàpies, la Fundació Joan Miró, el Gran Teatre del Liceu, el Museu d'Art Contemporani de Barcelona, el Palau de la Música y el Teatre Lliure.

Tabla 3 - Principales elementos de la titularidad de las instituciones culturales de Barcelona (2012)

\begin{tabular}{|l|l|l|l|l|}
\hline & \multicolumn{1}{|c|}{$\begin{array}{c}\text { Origen } \\
\text { iniciativa }\end{array}$} & $\begin{array}{l}\text { Titularidad } \\
\text { edificio }\end{array}$ & $\begin{array}{c}\text { Titularidad } \\
\text { gestión }\end{array}$ & $\begin{array}{c}\text { Titularidad } \\
\text { colección }\end{array}$ \\
\hline Auditori (AUD) & Sector público & $\begin{array}{l}\text { Sector } \\
\text { público }\end{array}$ & $\begin{array}{l}\text { Sector } \\
\text { público }\end{array}$ & \\
\hline $\begin{array}{l}\text { Centre de Cultura Contemporània de } \\
\text { Barcelona (CCCB) }\end{array}$ & Sector público & $\begin{array}{l}\text { Sector } \\
\text { público }\end{array}$ & $\begin{array}{l}\text { Sector } \\
\text { público }\end{array}$ & \\
\hline Fundació Antoni Tàpies (FAT) & Tercer sector & $\begin{array}{l}\text { Sector } \\
\text { público }\end{array}$ & Tercer sector & Tercer sector \\
\hline Fundació Joan Miró (FJM) & Tercer sector & Tercer sector & Tercer sector & Tercer sector \\
\hline Gran Teatre del Liceu (GTL) & Sector privado & $\begin{array}{l}\text { Sector } \\
\text { público }\end{array}$ & Mixta (1) & \\
\hline
\end{tabular}




\begin{tabular}{|l|l|l|l|l|}
\hline & \multicolumn{1}{|c|}{$\begin{array}{c}\text { Origen } \\
\text { iniciativa }\end{array}$} & $\begin{array}{l}\text { Titularidad } \\
\text { edificio }\end{array}$ & $\begin{array}{c}\text { Titularidad } \\
\text { gestión }\end{array}$ & $\begin{array}{l}\text { Titularidad } \\
\text { colección }\end{array}$ \\
\hline $\begin{array}{l}\text { Museu d'Art Contemporani de Barcelona } \\
\text { (MACBA) }\end{array}$ & Sector público & $\begin{array}{l}\text { Sector } \\
\text { público }\end{array}$ & Mixta & Tercer sector \\
\hline Mercat de les Flors (MF) & Sector público & $\begin{array}{l}\text { Sector } \\
\text { público }\end{array}$ & $\begin{array}{l}\text { Sector } \\
\text { público }\end{array}$ & \\
\hline $\begin{array}{l}\text { Museu Nacional d'Art de Catalunya } \\
\text { (MNAC) }\end{array}$ & Sector público & $\begin{array}{l}\text { Sector } \\
\text { público }\end{array}$ & $\begin{array}{l}\text { Sector } \\
\text { público }\end{array}$ & $\begin{array}{l}\text { Sector } \\
\text { público }\end{array}$ \\
\hline Museu Picasso de Barcelona (MPB) & Sector público & $\begin{array}{l}\text { Sector } \\
\text { público }\end{array}$ & $\begin{array}{l}\text { Sector } \\
\text { público }\end{array}$ & $\begin{array}{l}\text { Sector } \\
\text { público }\end{array}$ \\
\hline Palau de la Música (PM) & Tercer sector & Tercer sector & Mixta & \\
\hline Teatre Lliure (TLL) & Tercer sector & $\begin{array}{l}\text { Sector } \\
\text { público }\end{array}$ & Mixta & \\
\hline Teatre Nacional de Catalunya (TNC) & Sector público & $\begin{array}{l}\text { Sector } \\
\text { público }\end{array}$ & $\begin{array}{l}\text { Sector } \\
\text { público }\end{array}$ & \\
\hline
\end{tabular}

Fuente: elaboración propia

La titularidad de la gestión es uno de los elementos que generan una complejidad mayor en la caracterización de los equipamientos culturales de Barcelona. En diversos casos nos encontramos con arquitecturas institucionales complejas en las que existe un consorcio que gestiona el edificio y una fundación (una institución con personalidad jurídica privada) que gestiona toda o parte de la actividad (Martinez y Font, 2010) ${ }^{9}$.

No obstante, más allá de este criterio jurídico de la titularidad, uno de los elementos característicos del Modelo Barcelona y de sus instituciones culturales es la profusión de fórmulas de cooperación público-privadas en la gestión. Este sería el caso tanto del GTL, en el que el Consejo de Mecenazgo tiene una presencia activa en los órganos de gobierno, como del MACBA, que cuenta con la presencia de la Fundació Museu d'Art Contemporani en sus órganos de gobierno. Es también una característica fundamental del Palau de la Música, en la que el Orfeó Català es parte fundadora de la fundación que lo gestiona y propietaria del edificio. Finalmente, este carácter híbrido también sería aplicable al Teatre Lliure, cuyo ente de gestión propio es una fundación privada creada por profesionales del sector del teatro.

\section{Conclusiones}

Las industrias culturales se han convertido en los últimos treinta años en uno de los instrumentos privilegiados para impulsar la regeneración de las ciudades y para

9 Son los casos del Auditori, el Liceu y el Palau de la Música, en los que se consideró que una fundación era un tipo de organización que ofrecía más flexibilidad y agilidad para la gestión. No obstante, este tipo de arquitectura institucional ha sido puesta en duda por su opacidad, dificultad de control y falta de concordancia con los principios de gestión de los fondos públicos (Roselló y Rius, 2009). 
promover el desarrollo de la llamada creativa (Bianchini, 1993a, Scott, 2000, Scott, 2010). Por ello, las políticas culturales se han transformado en una herramienta clave para la gobernanza urbana, especialmente en ciudades postindustriales como es el caso de Barcelona (Degen y García, 2012). Esta instrumentalización de la política cultural hacia el desarrollo económico y social local (Gray, 2007) ha motivado el surgimiento de modelos de política cultural locales con una orientación emprendedora (Rodriguez Morató, 2008) que han convertido los equipamientos culturales en uno de sus principales instrumentos por su capacidad de impulsar procesos de regeneración urbana (Whitt, 1987) o de potenciar la imagen de las ciudad (Plaza, 1999, González, 2011).

Sin embargo, cabe preguntarse si es posible hablar de un modelo local de equipamiento cultural en correspondencia con el Modelo Barcelona de política cultural. Tal y como hemos podido ver en este estudio, en la génesis de los equipamientos culturales o en la inclusión de los equipamientos culturales en el sector público, el gobierno local ha ejercido un notable liderazgo, cumpliendo así su estrategia emprendedora desarrollada en otros campos de la actividad pública. En este proceso, el gobierno local ha implicado al resto de administraciones públicas, generalizando un esquema de gobernanza cultural multinivel. En segundo lugar, los equipamientos culturales han sido intensamente utilizados para la regeneración urbana como es el caso del Raval, Montjuïc o Glòries, creando así tres nuevos polos de actividad cultural. En tercer lugar, podemos ver cómo se ha potenciado la cooperación público-privada como una oportunidad de desarrollo de los equipamientos culturales, generando así unos equipamientos culturales híbridos (J. Rius, 2012).

No obstante, no todo el balance del Modelo Barcelona de política y equipamientos culturales es positivo. En primer lugar, porque el hecho de que se haya adoptado esta estrategia emprendedora ha comportado que en la ciudad de Barcelona no se haya realizado ningún plan de equipamientos culturales a partir de las necesidades ciudadanas y que estos se hayan planificado en función de la aparición de oportunidades estratégicas (Martinez y Font, 2010). Por otra parte, la mencionada voluntad de integración de diferentes administraciones públicas hace complejo el encaje de los diferentes objetivos y perspectivas de estas en la misión de un equipamiento cultural. Es por ello que se ha intentado desarrollar un instrumento de tutela y gobernanza como los contratos programa (J. Rius y Rubio, 2013). Asimismo, actualmente algunos de los máximos dirigentes de la política cultural consideran que las estructuras de gobernanza deberían racionalizarse para establecer cuál es la administración pública responsable de cada equipamiento cultural con el fin de garantizar su sostenibilidad en el marco de la reducción actual de los presupuestos públicos dedicados a la cultura (Mascarell, 2012).

En todo caso, actualmente el caso barcelonés muestra, por una parte, las relaciones entre el modelo de política cultural y el modelo de equipamiento cultural, y, por otra, cómo se han transformado las relaciones entre ciudad, administraciones públicas y sector cultural. De las grandes instituciones culturales generadas por los gobiernos nacionales de los estados-nación y orientadas a la reproducción de la alta cultura, hemos pasado a unas instituciones culturales que son funcionales al nuevo 
rol económico de la cultura como instrumento en la competición global de las ciudades (Scott, 2010). En este contexto, los equipamientos culturales se ven influidos por los modelos locales de desarrollo local y su política cultural, transformándose así su génesis, desarrollo y gestión, como hemos podido ver en el caso de Barcelona.

\section{Bibliografía}

Alier, Roger 1978, "El Liceu desde dentro y desde fuera", Revista Destino, 2109: 20-22.

Balibrea, Mari Paz 2001, "Urbanism, culture and the post-industrial city: Challenging the 'Barcelona model'", Journal of Spanish Cultural Studies, 2: 187-210.

Belfiore, Eleonora 2004, "Auditing Culture. The subsidised cultural sector in the New Public Management", International Journal of Cultural Policy, 10: 183202.

Belfiore, Eleonora 2002, "Art as a means of alleviating social exclusion: Does it really work? A critique of instrumental cultural policies and social impact studies in the UK", International Journal of Cultural Policy, 8: 91-106.

Belfiore, Eleonora y Bennett, Oliver 2007, "Rethinking the social impact of the arts", International Journal of Cultural Policy, 13: 135-151.

Bianchini, Franco 1993a, "Remaking European Cities: the role of cultural policies" en Franco Bianchini y Michael Parkinson, eds.Cultural Policy and Urban Regeneration: the West European Experience. Manchester: Manchester University Press, , pp. 1-19.

Bianchini, Franco 1993b, Urban Cultural Policy in Britain and Europe: Towards Cultural Planning. London: Institute for Cultural Policy Studies.

Blanco, Ismael 2009a, "Revista Española de Ciencia Politica", Gobernanza urbana y políticas de regeneración: el caso de Barcelona, 20: 125-146.

Blanco, Ismael 2009b, "Does a Barcelona Model Really Exist? Periods, Territories and Actors in the Process of Urban Transformation", Local Government Studies, 35: 355-369.

Caminal, Josep 2004, "El Liceu avisa que tocará techo", La Vanguardia, 20/02/2004: 38.

Cherbo, Joni M. y Wyszomirski, Margaret J. 2000, The Public Life of the Arts in America. Brunswick: Rutgers.

Connolly, Mark Gerard 2011, "The 'Liverpool model(s)': cultural planning, Liverpool and Capital of Culture 2008", International Journal of Cultural Policy, : 120.

Coppinger, Nathalie, Joder, Mélanie, Leca, Charlotte y Dupuit, Jean-Sébastien 2007, Rapport sur la tutelle et le pilotage des opérateurs au ministère de la culture. Paris: Ministère de l'économie, des finances et de l'industrie.

Currid, Elizabeth 2007, The Warhol economy: how fashion, art, and music drive New York City: Princeton University Press. 
Degen, Mónica y García, Marisol 2012, "The Transformation of the Barcelona Model?: An Analysis of Culture, Urban Regeneration and Governance", International Journal of Urban and Regional Research, 36: 1022-1038.

Department of Culture, Media and Sport 2010, Annual Report 2009. London: Department of Culture, Media and Sport.

DEPS 2011, Culture and Medias. Prospective de politiques culturelles. Paris: Ministère de la Culture - La documentation Française.

Dewey, Patricia 2004, "From arts management to cultural administration", International Journal of Arts Management, 6: 13-22.

Esping-Andersen, Gosta 1993, Los tres mundos del Estado de Bienestar. València: Alfons el Magnànim.

Fedeli, Silvia y Santoni, Michele 2006, "The Government's Choice of Bureaucratic Organisation: An Application to Italian State Museums", Journal of Cultural Economics, 30: 41-72.

Font, Jordi 1991, Papers de política cultural. Barcelona: Edicions 62.

García, Beatriz 2004, "Urban regeneration, arts programming and major events: Glasgow 1990, Sydney 2000 and Barcelona 2004 ", International Journal of Cultural Policy, 10: 103-118.

Gattinger, Monica y Saint-Pierre, Diane 2008, "Can national cultural policy approaches be used for sub-national comparisons? An analysis of the Québec and Ontario experiences in Canada", International Journal of Cultural Policy, 14: 335-354.

Generalitat de Catalunya 2010, Pressupostos de la Generalitat de Catalunya 2010. Barcelona: Generalitat de Catalunya.

González, Sara 2011, "Bilbao and Barcelona ‘in Motion’. How Urban Regeneration 'Models' Travel and Mutate in the Global Flows of Policy Tourism", Urban Studies, 48: 1397-1418.

Gray, Clive 2008, "Instrumental policies: causes, consequences, museums and galleries", Cultural Trends, 17: 209-222.

Gray, Clive 2007, "Commodification and instrumentality in cultural policy", International Journal of Cultural Policy, 13: 203-215.

Guillemat, Eslisabeth 2008, "El Plan Estratégico de cultura de Barcelona 2007-2015: nuevos acentos 2006" en Felix Manito, ed.Planificación estratégica de la cultura en España. Madrid: Fundación Autor, , pp. 189-218.

Harvey, David 1989, "From Managerialism to Entrepreneurialism: The Transformation in Urban Governance in Late Capitalism", Geografiska Annaler.Series B, Human Geography, 71: 3-17.

ICUB 2006, Nous accents 2006. Pla Estrategic del Sector Cultural de Barcelona. Barcelona: Ajuntament de Barcelona.

ICUB 1999, Bcn, Accent de Cultura. Pla Estratègic del Sector Cultural de la Ciutat. Barcelona: Ajuntament de Barcelona.

Landry, Charles y Bianchini, Franco 1995, The creative city. Great Britain: Demos. Lord, Barry y Lord, Gael 1998, Manual de gestión de museos. Barcelona: Ariel. 
Majoor, Stan 2011, "Framing Large-Scale Projects: Barcelona Forum and the Challenge of Balancing Local and Global Needs", Journal of Planning Education and Research, 31: 143-156.

Maragall, Pascual 1989, "Prefaci" en Josep Maria Flotats, ed.Un projecte per al Teatre Nacional. Barcelona: Edicions de la Revista de Catalunya, , pp. 3-15.

Marshall, Tim 2000, "Urban Planning and Governance: Is there a Barcelona Model?", International Planning Studies, 5: 299-319.

Marshall, Tim 1996, "Barcelona fast forward? City entrepreneurialism in the 1980s and 1990s", European Planning Studies, 4: 147-165.

Martinez, Santi \& Font, Josep 2010, "Infraestructures públiques al servei de la creació i de la comunitat" en Gescenic, ed.L'avaluació externa de projectes culturals, pp. 37-49.

Martorella, Roseanne 1982, The Sociology of Opera. New York: Bergin Publishers.

Mascarell, Ferran 2012, "Compareixènça de l'Honorable Conseller de Cultura Ferran Mascarell", Comissió de Cultura del Parlament de Catalunya, 11: 9-37.

Menger, Piere-Michel 2010, Cultural policies in Europe. From a state to a citycentered perspective on cultural generativity. Tokyo: National Graduate Institute for Policy Studies.

Ministerio de Economía y Hacienda 2009, Presupuestos generales del Estado 2009: Ministerio de Cultura. Madrid: Ministerio de Economía y Hacienda.

Moulin, Raymond 1992, L'artiste, l'institution et le marché. Paris: Flammarion.

Mulcahy, Kevin 1998, "Cultural Patronage in Comparative Perspective: Public Support for the Arts in France, Germany, Norway, and Canada", Journal of Arts Management, Law and Society, 27: 247-263.

Plaza, Beatriz 1999, "The Guggenheim-Bilbao Museum Effect: A reply", International Journal of Urban and Regional Research, 23: 589-592.

Rius, Joaquim 2012, "Política cultural e hibridación de las instituciones culturales. El caso de Barcelona", Revista Española de Ciencia Política, 29: 85-105.

Rius, Joaquim y Rubio, Arturo 2013, "The governance of national cultural organisations: comparative study of performance contracts with the main cultural organisations in England, France and Catalonia", International Journal of Cultural Policy, 19: 249-269.

Rodriguez Morató, Arturo 2008, "La emergencia de una capital cultural europea" en Mónica Degen y Soledad García, eds.La metaciudad: Barcelona. Transformación de una metropolis. Barcelona: Antrophos, .

Rodriguez Morató, Arturo 2005, "La reinvención de la política cultural a escala local: el caso de Barcelona", Sociedade e Estado, 20: 351-376.

Roselló, David y Rius, Joaquim 2009, La cooperació entre ateneus i ens locals per una cultura del segle XXI. Barcelona: Departament de Cultura i Mitjans de Comunicació.

Rubio Arostegui, Juan Arturo 2008, "Génesis configuración y evolución de la política cultural del estado a través del Ministerio de Cultura.: 1977-2007", RIPS: Revista de Investigaciones Políticas y Sociológicas, 7: 55-70. 
Rubio, Arturo y Rius, Joaquim 2012, "La modernización de la gestión pública de la cultura. Análisis comparado del caso de los equipamientos culturales de las comunidades autónomas de Cataluña y Madrid", Gestión y Análisis de Políticas Públicas, 8: 79-92.

Schuster, J. Mark 1998, "Neither Public Nor Private: The Hybridization of Museums", Journal of Cultural Economics, 22: 127-150.

Scott, Allen 2010, "Cultural economy and the creative field of the city", Geografiska Annaler: Series B, Human Geography, 92: 115-130.

Scott, Allen 2000, The Cultural Economy of Cities. London [etc.]: Sage in association with Theory, Culture \& Society. Nottingham Trent University.

Subirats, Joan y Rius, Joaquim 2008, Del Xino Al Raval. Cultura i transformació social a la Barcelona central. Barcelona: Editorial Hacer.

Subirós, Josep 1999, Estrategias culturales y renovación urbana. Barcelona: Aula Barcelona.

Subirós, Josep 1998, El vol de la fletxa. Barcelona'92: Crònica de la reinvenció de la ciutat. Barcelona: Electa.

Turisme de Barcelona 2012, Activitat turistica 1993/2011. Barcelona: Turisme de Barcelona.

Urfalino, Philippe 1996, L'invention de la politique culturelle. Paris: La Documentation Française.

Villarroya, Anna 2012, "Cultural policies and national identity in Catalonia", International Journal of Cultural Policy, 18: 31-45.

Whitt, J. Allen 1987, "Mozart in the Metropolis: The Arts Coalition and the Urban Growth Machine", Urban Affairs Review, 23: 15-36.

Zimmer, Annette y Toepler, Stefan 1999, "The Subsidized Muse: Government and the Arts in Western Europe and the United States", Journal of Cultural Economics, 23: 33-49.

Zimmer, Annette y Toepler, Stefan 1996, "Cultural Policies and the Welfare State: The Cases of Sweden, Germany, and the United States", The Journal of Arts Management, Law and Society, 26: 167-193. 